\title{
Urinary isoflavonoids and risk of type 2 diabetes: a prospective investigation in US women
}

\author{
Ming Ding ${ }^{1}$, Adrian A. Franke ${ }^{2}$, Bernard A. Rosner ${ }^{3,4}$, Edward Giovannucci ${ }^{1,3}$, Rob M. van Dam ${ }^{1,5}$, \\ Shelley S. Tworoger ${ }^{3,6}$, Frank B. Hu ${ }^{1,3,6}$ and Qi Sun ${ }^{1,3 *}$ \\ ${ }^{1}$ Department of Nutrition, Harvard School of Public Health, Boston, MA 02115, USA \\ ${ }^{2}$ Department of Food Science and Human Nutrition, College of Tropical Agriculture and Human Resources, University of \\ Hawai'i Cancer Center, Honolulu, HI 96813, USA \\ ${ }^{3}$ Channing Division of Network Medicine, Brigham and Women's Hospital and Harvard Medical School, Boston, MA O2115, USA \\ ${ }^{4}$ Department of Statistics, Harvard School of Public Health, Boston, MA 02115, USA \\ ${ }^{5}$ Department of Epidemiology and Public Health, Saw Swee Hock School of Public Health and Yong Loo Lin School of Medicine, \\ National University of Singapore and National University Health System, Singapore 117597 \\ ${ }^{6}$ Department of Epidemiology, Harvard School of Public Health, Boston, MA 02115, USA
}

(Submitted 21 February 2015 - Final revision received 2 July 2015 - Accepted 5 August 2015 - First published online 15 September 2015)

\section{Abstract}

To examine the association between urinary excretion of isoflavonoids and risk of type 2 diabetes (T2D), we conducted a nested case-control study among 1111 T2D pairs identified during 1995-2008 in the Nurses' Health Study (NHS) and NHSII, who were free of diabetes, CVD and cancer at urine sample collection. Urinary excretion of daidzein and genistein, as well as their metabolites $O$-desmethylangolensin (O-DMA), dihydrogenistein (DHGE) and dihydrodaidzein (DHDE) was assayed using liquid chromatography MS. Self-reported T2D incident cases were confirmed using a validated questionnaire. Higher urinary excretion of daidzein and genistein was associated with a lower risk of T2D in the combined cohorts. Comparing extreme tertiles of the urinary markers, the OR of T2D were $0.71(95 \% \mathrm{CI} 0.55,0.93)$ for daidzein and $0.74(95 \% \mathrm{CI} 0.56,0.97)$ for genistein, although the test for linear trend was not significant for genistein $\left(P_{\text {trend }}=0.03\right.$ and 0.15 , respectively). DMA, DHDE and DHGE were non-significantly associated with a lower T2D risk. The inverse association of daidzein with T2D risk was stronger among post-menopausal women who did not use hormone replacement therapy $\left(P_{\text {interaction }}=0.001\right)$ : the OR was 0.58 (95\% CI 0.34, 0.97) comparing extreme tertiles among these women. In conclusion, urinary excretion of isoflavones was associated with a lower T2D risk in US women, especially among post-menopausal women who did not use hormone. Further research is warranted to replicate these observations among western populations with similarly low overall isoflavone intake.

Key words: Isoflavones: Diabetes: Menopausal status: Nested case-control studies

Type 2 diabetes (T2D) is a chronic disease with an increasing prevalence worldwide. The total number of diabetes patients is estimated to reach 592 million globally by the year $2035^{(1)}$. Excessive body weight, unhealthy diet, lack of exercise and smoking are major risk factors of $\mathrm{T} 2 \mathrm{D}^{(2)}$. Seeking effective dietary and lifestyle measures for T2D prevention has been a priority to counteract the increasing prevalence and incidence of diabetes ${ }^{(3-5)}$. Women may be at a particularly high risk at middle life when menopause and ageing jointly increase the risk of $\mathrm{T} 2 \mathrm{D}^{(6-8)}$. Large clinical trials and prospective cohort studies have consistently shown that hormone replacement therapy may reduce the risk of T2D in post-menopausal women ${ }^{(9-12)}$. However, it is unclear whether natural phytoestrogens, such as isoflavones, may be associated with T2D risk.

Clinical trials showed that isoflavone supplements did not improve glucose control ${ }^{(13,14)}$, although these clinical trials were limited by small sample size and short duration of follow-up. Several cohort studies have evaluated the association of isoflavone intake assessed using FFQ with risk of T2D, and mixed results were observed ${ }^{(15-17)}$. One potential reason for the inconsistent findings in the observational studies may lie in the difficulties of using FFQ to assess isoflavone intakes. Soya foods are the main source of isoflavone intakes, although other foods contain various amounts of isoflavones as well ${ }^{(18)}$. Isoflavone intake estimated from FFQ is thus subject to measurement errors $^{(19)}$, especially in Western populations who infrequently consume soya foods. In addition, isoflavone intake estimated from FFQ does not take into account inter-individual variations in bioavailability ${ }^{(20)}$. In addition, FFQ cannot be used to estimate the gut microbiota metabolites of isoflavones - including $O$-desmethylangolensin $(O-D M A)^{(21)}$, dihydrogenistein (DHGE) and dihydrodaidzein (DHDE) - and equol ${ }^{(22)}$, which may exert

Abbreviations: DHDE, dihydrodaidzein; DHGE, dihydrogenistein; O-DMA, O-desmethylangolensin; NHS, Nurses' Health Study; T2D, type 2 diabetes.

* Corresponding author: Q. Sun, email qisun@hsph.harvard.edu 
biological effects in addition to their parent compounds (i.e. daidzein and genistein) ${ }^{(23)}$. In this regard, use of isoflavone metabolites in blood or urine as objective markers of isoflavone intake is an appealing approach ${ }^{(24,25)}$.

In the current investigation, we utilised data from a combined cohort based on two well-characterised cohorts of US women, the Nurses' Health Study (NHS) and NHSII, to prospectively evaluate the association of urinary excretion of isoflavone metabolites with risk of T2D. We also examined the hypothesis that isoflavone excretion may especially be associated with lower T2D risk among post-menopausal women who do not receive replacement therapy and thus have a low exposure to exogenous estrogens.

\section{Methods \\ Study population}

The NHS began in 1976, when 121700 female registered nurses aged 30-55 years residing in eleven states were enroled and completed a baseline questionnaire about their lifestyle and medical history. The NHSII was established in 1989 and consisted of 116430 younger female registered nurses aged 25-42 years at baseline. These nurses also responded to a baseline questionnaire similar to that of the NHS. In both cohorts, questionnaires were collected at baseline and biennially thereafter, to update information on age, weight, smoking status, physical activity, medication use, menopausal status, post-menopausal hormone use and disease status including hypertension, hypercholesterolaemia, CVD and cancer.

\section{Urine sample collection}

A total of 18743 NHS participants aged 53 to 80 years provided morning spot urine samples from 2000 to 2002, and 29611 NHSII participants aged 32 to 52 years provided morning spot urine samples from 1996 to 1999. For both cohorts, the samples were returned to a central biorepository Via overnight courier and were immediately processed upon arrival and aliquoted into cryotubes, which were stored in the vapour phase of liquid nitrogen freezers at $\leq-130^{\circ} \mathrm{C}$. Loss to follow-up was $<10 \%$ among participants who provided blood and urine samples.

\section{Prospective case-control study design}

We conducted a prospective, nested case-control study among participants who provided urine samples and were free of selfreported diabetes, CVD and cancer at urine collection in NHS and NHSII. T2D cases diagnosed within the 1st year since urine sample collection were excluded from selection in order to reduce the potential for reverse causation bias. During follow-up from urine collection through 2008 (NHS)/2007 (NHSII), we prospectively identified and confirmed 1111 T2D cases (NHS: 456; NHSII: 655) and randomly selected one control from diabetes-free participants using risk set sampling ${ }^{(26)}$ The cases and controls were matched for age at sample collection, month of sample collection, fasting status ( $\geq 8 \mathrm{~h}$ or not), first morning urine (yes or no) and race (White or other races) in both cohorts. In NHSII, we additionally matched for menopausal status (yes, no), luteal day of the menstrual cycle (date of next period minus date of sample collection) for premenopausal women and hormone replacement therapy (yes or no) for post-menopausal women. The study protocol was approved by the institutional review board of Brigham and Women's Hospital and the Human Subjects Committee Review Board of Harvard School of Public Health.

\section{Ascertainment of type 2 diabetes}

We sent a validated supplemental questionnaire to those who reported a physician diagnosis of $\mathrm{T} 2 \mathrm{D}$ to confirm the incidence $^{(27)}$. We used at least one of the following American Diabetes Association 1998 criteria to confirm self-reported T2D diagnosis: an elevated glucose concentration (fasting plasma glucose $\geq 7.0 \mathrm{mmol} / 1$, random plasma glucose $\geq 11.1 \mathrm{mmol} / \mathrm{l}$, or plasma glucose $\geq 11 \cdot 1 \mathrm{mmol} / 1$ after an oral glucose load) and at least one symptom related to diabetes; no symptoms but elevated glucose concentrations on two separate occasions; or treatment with insulin or oral hypoglycaemic medication. Only confirmed T2D cases were included in the current study.

\section{Assessment of diet}

Validated FFQ have been administered since 1984 in NHS and since 1991 in $\mathrm{NHSII}^{(28)}$. Similar FFQ were subsequently sent to participants every 2-4 years to update diet. In these FFQ, we enquired about the consumption frequency of 118-166 food items in the past year, and how often (from 'never or less than once per month' to 'six or more times per day') on an average they consumed each food item of a standard portion size. Major soya foods, such as tofu and soya milk, have been simultaneously included on the FFQ since 1998 in the NHS and since 1999 in the NHSII. An overall measure of diet quality was calculated using the Alternate Healthy Eating Index (AHEI) score, excluding the soya food items, such as tofu and soya milk ${ }^{(29)}$. AHEI is based on foods and nutrients that are predictive of the risk of developing major chronic diseases. To calculate AHEI score, we assigned individual score to each of the food group and summed up the scores, with a higher score indicative of better diet quality ${ }^{(30)}$.

\section{Laboratory measurements}

In the current study, we used electrospray ionisation liquid chromatography MS to measure isoflavonoids in urine samples, which has been validated except for the use of an orbitrap mass spectrometer ${ }^{(21,31)}$. Urinary creatinine levels were measured using a Roche-Cobas MiraPlus clinical chemistry autoanalyzer (Roche Diagnostics). The average intra-assay CV was $4.1 \%$ for daidzein, $7 \cdot 6 \%$ for genistein, $8 \cdot 2 \%$ for DHDE, $10 \cdot 1 \%$ for DHGE, $8.1 \%$ for O-DMA and $5.6 \%$ for creatinine. We calculated creatinine-adjusted concentrations ( $\mathrm{nmol} / \mathrm{g}$ creatinine) of isoflavonoids by dividing the isoflavonoid levels (nmol/l) by creatinine levels $(\mathrm{g} / \mathrm{l})$. In a pilot study that evaluated withinperson stability of the isoflavonoids, intra-class correlation coefficients (ICC) of two urine samples from 58 NHSII participants 
collected 1-2 years apart were 0.05 for daidzein, 0.14 for genistein, $0 \cdot 16$ for DHDE, $0 \cdot 14$ for DHGE and 0.20 for $O$-DMA.

\section{Statistical methods}

We calculated Spearman correlation coefficients between urine excretions of isoflavone metabolites with soya foods, such as tofu and soya milk, estimated from FFQ. We adjusted for total energy intake $(\mathrm{kJ} / \mathrm{d}(\mathrm{kcal} / \mathrm{d})), \mathrm{BMI}\left(\mathrm{kg} / \mathrm{m}^{2}\right)$, physical activity (MET-h/week), age (years), smoking (never, past, current) and first morning urine (yes or no). This analysis was conducted among controls to facilitate comparison between cohorts.

We categorised the isoflavone biomarkers into tertiles. We used conditional logistic regression stratified by matching factors to model the association between isoflavone metabolites and risk of $\mathrm{T} 2 \mathrm{D}$ in the main analysis ${ }^{(26)}$. We additionally adjusted for hypertension at baseline (yes or no), hypercholesterolaemia at baseline (yes or no), BMI $\left(\mathrm{kg} / \mathrm{m}^{2}\right)$, smoking (non-smoker, past smoker, current smoker), AHEI score, physical activity (MET-h/week), total energy intake $(\mathrm{kJ} / \mathrm{d}(\mathrm{kcal} / \mathrm{d}))$, menopausal status (pre-menopausal or post-menopausal) (NHS only) and hormone replacement therapy (yes or no) (NHS only). $P$ values for linear trend were calculated by examining an ordinal score based on the median value in each tertile of isoflavones biomarker levels in the multivariate models.

Given that menopausal status and post-menopausal hormone therapy were not matching factors in NHS, we conducted stratified analyses by menopausal status and post-menopausal hormone therapy (yes $v$. no) using unconditional logistic regression to maximise statistical power. $P$ values for interactions were evaluated using the likelihood ratio test, comparing the multivariate model with and without interaction terms of dichotomised isoflavones and potential effect modifiers in conditional logistic regression. Joint associations of the urinary biomarkers and potential effect modifiers were estimated using conditional logistic regression. All $P$ values were two-sided. Data were analysed with the statistical analysis systems software package, version 9.3 (SAS Institute Inc.).

\section{Results}

Baseline characteristics of study participants are shown in Table 1. Compared with controls, T2D cases consumed a less healthful diet, engaged in less physical activity, had a higher BMI and were more likely to have a history of hypertension and hypercholesterolaemia. The mean value of the urine isoflavones was $3863 \mathrm{nmol} / \mathrm{g}$ creatinine among cases and $5435 \mathrm{nmol} / \mathrm{g}$ creatinine among controls. The baseline characteristics according to urine isoflavone excretion are shown in online Supplementary Table S1. Higher urinary isoflavone excretion was correlated with a healthier dietary pattern and higher levels of physical activity. We further compared the baseline characteristics of controls with that of the population, and the controls were comparable to the total participants in NHS and NHSII (online Supplementary Table S2).

Moderate to strong correlations among urinary isoflavone metabolites were observed (correlation coefficient: $0 \cdot 26-0 \cdot 79$ ), with the strongest correlation observed between daidzein and genistein (online Supplementary Table S3). Weak yet significant correlations were found between soya foods estimated from FFQ and urinary isoflavone excretion among controls.

Urinary excretion of total isoflavones was not associated with risk of T2D $\left(P_{\text {trend }}=0 \cdot 20\right)$. Higher levels of daidzein and genistein, which are the dominant metabolites of isoflavones, were associated with a lower risk of T2D (Table 2). For daidzein, compared with the lowest group, the OR of T2D were 0.78 (95\% CI $0.60,1.02)$ in the second tertile and 0.71 (95\% CI 0.55 , $0.93)$ in the highest tertile $\left(P_{\text {trend }}=0.03\right)$; for genistein, compared with the lowest group, the OR of T2D were 0.70 (95\% CI $0.53,0.93)$ in the second tertile and $0.74(95 \% \mathrm{CI} 0.56,0.97)$ in the highest tertile $\left(P_{\text {trend }}=0 \cdot 15\right)$. O-DMA, DHDE and DHGE which are the metabolites of daidzein and genistein - were nonsignificantly associated with a lower risk of T2D. Comparing the extreme tertiles, the OR of T2D were 0.92 (95\% CI $0.70,1.21$ ) for O-DMA, 0.80 (95\% CI 0.60, 1.06) for DHDE and 0.82 (95\% $0.62,1.08)$ for DHGE. Further adjustment of individual dietary components - such as red meats, fruits and vegetables, and coffee - in replacement of AHEI did not change the results materially. In a sensitivity analysis, we further excluded 945 participants whose $\mathrm{HbA}_{1 \mathrm{c}}$ levels were $\geq 5.7 \%$ and repeated the analysis. The inverse association between daidzein and risk of T2D persisted: compared with the lowest tertile, the OR of T2D were $0.91(95 \%$ CI $0.47,1.77)$ in the second tertile and 0.49 (95\% CI 0.26, 0.94) in the highest tertile $\left(P_{\text {trend }}=0.01\right)$.

Stratified analyses by menopausal status and hormone use were conducted. Significant interaction by post-menopausal hormone use was found for daidzein $\left(P_{\text {interaction }}=0.001\right)$ (Table 3). Comparing extreme tertiles of the urinary daidzein, the OR of T2D were 0.81 (95\% CI $\left.0.54,1.21 ; P_{\text {trend }}=0.41\right)$ for pre-menopausal women, 0.76 (95\% CI 0.52, $\left.1.11 ; P_{\text {trend }}=0.19\right)$ for post-menopausal women with hormone use and $0.58(95 \%$ CI 0.34, 0.97; $P_{\text {trend }}=0.06$ ) for post-menopausal women without hormone use. Consistently, in joint association analysis, the inverse association between urinary daidzein and risk of T2D appeared to be stronger in post-menopausal women without hormone use (Fig. 1(a)). No significant interactions between other metabolites and risk of T2D by menopausal status and hormone use were found. Further stratified analyses were conducted by age, BMI and AHEI, and the inverse association appeared to be more apparent among women with a BMI $<30$ $\left(P_{\text {interaction }}=0.03\right)$ (online Supplementary Table S4).

\section{Discussion}

In these two cohorts of US women, urinary excretion of the main isoflavone metabolites, daidzein and genistein, was associated with a lower risk of T2D. Further analyses suggested that inverse association between daidzein and risk of T2D appeared to be stronger among post-menopausal women who did not take hormone therapy at sample collection. These associations were independent of established diabetes risk factors - such as BMI, physical activity and overall diet quality.

Isoflavones are able to bind to the oestrogen receptors (ER), especially ER- $\beta$, with $10^{3}-10^{4}$ less potency than oestradiol ${ }^{(23)}$. These compounds can exert either oestrogenic or anti-oestrogenic action depending on the level of oestradiol in circulation. When 
Table 1. Age-adjusted baseline characteristics according to diabetes cases and controls in the combined cohort (Medians and interquartile ranges; percentages)

\begin{tabular}{|c|c|c|c|c|}
\hline \multirow[b]{2}{*}{ Characteristics* $^{*}$} & \multicolumn{2}{|c|}{ Cases $(n 1111)$} & \multicolumn{2}{|c|}{ Controls ( $n$ 1111) } \\
\hline & Median & Interquartile range & Median & Interquartile range \\
\hline Age at urine collection (years) & 53.4 & & $53 \cdot 4$ & \\
\hline $\mathrm{BMI}\left(\mathrm{kg} / \mathrm{m}^{2}\right)$ & $32 \cdot 0$ & & $25 \cdot 9$ & \\
\hline Physical activity (MET-h/week) & $16 \cdot 4$ & & $19 \cdot 5$ & \\
\hline Current smoker (\%) & \multicolumn{2}{|c|}{11} & \multicolumn{2}{|c|}{7} \\
\hline Hypertension (\%) & \multicolumn{2}{|c|}{38} & \multicolumn{2}{|c|}{19} \\
\hline Hypercholesterolaemia (\%) & \multicolumn{2}{|c|}{55} & \multicolumn{2}{|c|}{35} \\
\hline Family history of diabetes (\%) & \multicolumn{2}{|c|}{37} & \multicolumn{2}{|c|}{21} \\
\hline First morning urine $(\%) \dagger$ & \multicolumn{2}{|c|}{88} & \multicolumn{2}{|c|}{88} \\
\hline Post-menopausal (\%)† & \multicolumn{2}{|c|}{83} & \multicolumn{2}{|c|}{83} \\
\hline $\begin{array}{l}\text { Post-menopausal hormone use ( } \% \text { of post-menopausal } \\
\text { women) } \dagger\end{array}$ & \multicolumn{2}{|c|}{43} & \multicolumn{2}{|c|}{43} \\
\hline White $(\%) \dagger$ & \multicolumn{2}{|c|}{96} & \multicolumn{2}{|c|}{97} \\
\hline \multicolumn{5}{|l|}{ Urinary metabolites (nmol/g creatinine) } \\
\hline Daidzein & 311 & 112,956 & 343 & 123,1013 \\
\hline Genistein & 122 & 48,424 & 128 & 48,419 \\
\hline Desmethylangolensin & 29 & 9,116 & 36 & 12,135 \\
\hline Dihydrodaidzein & 34 & 6,217 & 42 & 9,288 \\
\hline Dihydrogenistein & 25 & 10,72 & 27 & 12,78 \\
\hline \multicolumn{5}{|l|}{ Diet } \\
\hline Total energy $(\mathrm{kJ} / \mathrm{d})$ & 7803 & & 7426 & \\
\hline Total energy (kcal/d) & 1865 & & 1775 & \\
\hline Alcohol $(g / d)$ & 0.3 & & 0.4 & \\
\hline Coffee (cups/d) & $1 \cdot 0$ & & 1.2 & \\
\hline Soft drinks (servings/d) & 1.2 & & 0.9 & \\
\hline Tofu consumers (\%) & \multicolumn{2}{|c|}{10} & \multicolumn{2}{|c|}{12} \\
\hline Soya milk consumers (\%) & \multicolumn{2}{|c|}{4} & \multicolumn{2}{|r|}{7} \\
\hline Fruits (servings/d) & $2 \cdot 0$ & & $2 \cdot 0$ & \\
\hline Vegetables (servings/d) & 3.5 & & 3.3 & \\
\hline Red meat (servings/d) & 1.5 & & 1.3 & \\
\hline Fish (servings/d) & 0.2 & & 0.2 & \\
\hline Hot dogs (servings/d) & 0.1 & & 0.1 & \\
\hline Alternate Healthy Eating Index score & 49 & & 52 & \\
\hline
\end{tabular}

NHS, Nurses' Health Study.

* All of the variables are age-adjusted, except the urinary metabolites of isoflavones. Values of continuous variables are medians. Percentages are based on non-missing data.

† Matching factors; menopausal status and hormone replacement therapy are matching factors for NHSII only.

endogenous oestrogen levels are low, isoflavones primarily exert oestrogen-like effects ${ }^{(32)}$. Our observation that a stronger inverse association between isoflavones - especially daidzein - and risk of T2D was noted among post-menopausal women without current hormone use is in line with the notion that isoflavones exert oestrogen-like effects on blood glucose when circulatingoestrogen levels are low. In addition, this inverse association was also consistent with the results from short-term clinical trials. Supplementation of isoflavones did not significantly lower fasting glucose or insulin levels in a comprehensive meta-analysis of randomised clinical trials ${ }^{(13)}$. The non-significant results of those trials might be because of short duration and might not reflect the potential anti-diabetic effect of long-term isoflavone intake. Meanwhile, in another meta-analysis that focused on perimenopausal and post-menopausal non-Asian women who did not take hormone replacement therapy, isoflavone supplementation significantly lowered fasting insulin and homoeostasis model assessment of insulin resistance levels ${ }^{(33)}$. Meanwhile, a stronger inverse association between urinary daidzein and risk of T2D was observed among non-obese participants. This association may be related to concentration of blood-circulating oestrogen $^{(34)}$, as adipose tissue is the main source of circulating estrogens for post-menopausal women without hormone use $\mathrm{e}^{(35)}$.
Besides binding to ER, isoflavones may also bind to and activate nuclear receptors, which regulate lipid and glucose metabolism, including liver $\mathrm{X}$ binding receptors, sterol regulated element binding protein, peroxisome-proliferator activated receptors $\alpha(\operatorname{PPAR} \alpha)$ and $\operatorname{PPAR} \gamma^{(36-38)}$. Isoflavones may also increase the phosphorylation of AMP-activated protein kinase and acetyl-CoA carboxylase to improve glucose up-take and fatty acid oxidation ${ }^{(39)}$. Animal studies have also shown that isoflavones may improve hyperglycaemia, glucose tolerance and circulating insulin concentrations ${ }^{(40)}$.

Several cohort studies have been conducted to examine the association between intakes of soya foods and isoflavones estimated using FFQ and risk of T2D ${ }^{(15-17,41,42)}$. For example, in a Japanese population, higher intakes of isoflavones were not associated with diabetes risk in the total study population, but an inverse association was found among overweight women $^{(16)}$. In the Singapore-Chinese Health Study, intakes of total isoflavones were not associated with T2D risk, probably because many soya foods were sweetened in Singapore ${ }^{(15)}$. In the EPIC-InterAct Study, total isoflavone intake was not associated with T2D risk among men and women in eight European countries $^{(17)}$. In addition, two other studies assessed soya food consumption with risk of T2D. An inverse association 
Table 2. Odds ratios of type 2 diabetes by tertiles of urinary isoflavones ( $\mathrm{nmol} / \mathrm{g}$ creatinine) in the combined cohort (Odds ratios and $95 \%$ confidence intervals; medians and ranges)

\begin{tabular}{|c|c|c|c|c|c|c|}
\hline & \multicolumn{5}{|c|}{ Tertiles of urinary markers } & \multirow[b]{3}{*}{$P_{\text {trend }}$} \\
\hline & \multirow[b]{2}{*}{1 (lowest) } & \multicolumn{2}{|c|}{2} & \multicolumn{2}{|c|}{3 (highest) } & \\
\hline & & OR & $95 \% \mathrm{Cl}$ & OR & $95 \% \mathrm{Cl}$ & \\
\hline \multicolumn{7}{|l|}{ Daidzein } \\
\hline Median & 77 & \multicolumn{2}{|c|}{326} & \multicolumn{2}{|c|}{1529} & \\
\hline Range & 0,171 & \multicolumn{2}{|c|}{171,665} & \multirow{2}{*}{\multicolumn{2}{|c|}{665,196860}} & \\
\hline Case/control & $386 / 354$ & \multicolumn{2}{|c|}{$369 / 372$} & $356 / 385$ & & \\
\hline Model $1^{*}$ & 1.00 & 0.88 & $0.72,1.07$ & 0.85 & $0.70,1.04$ & 0.18 \\
\hline Model $2 \dagger$ & 1.00 & 0.78 & $0.60,1.02$ & 0.71 & $0.55,0.93$ & 0.03 \\
\hline \multicolumn{7}{|l|}{ Genistein } \\
\hline Median & 33 & \multicolumn{2}{|c|}{$\begin{array}{c}125 \\
65,269\end{array}$} & \multicolumn{2}{|c|}{729} & \\
\hline Range & 0,65 & \multirow{2}{*}{\multicolumn{2}{|c|}{$\begin{array}{l}65,269 \\
370 / 371\end{array}$}} & \multirow{2}{*}{\multicolumn{2}{|c|}{$\begin{array}{c}270,375579 \\
365 / 376\end{array}$}} & \\
\hline Case/control & $376 / 364$ & & & & & \\
\hline Model $1^{*}$ & 1.00 & 0.94 & $0.76,1.15$ & 0.92 & $0.74,1.13$ & 0.50 \\
\hline Model $2 \dagger$ & 1.00 & 0.70 & $0.53,0.93$ & 0.74 & $0.56,0.97$ & 0.15 \\
\hline \multicolumn{7}{|c|}{ Desmethylangolensin } \\
\hline Median & 6 & \multicolumn{2}{|c|}{33} & \multicolumn{2}{|c|}{225} & \\
\hline Range & 0,16 & \multirow{2}{*}{\multicolumn{2}{|c|}{$\begin{array}{c}16,78 \\
365 / 376\end{array}$}} & \multicolumn{2}{|c|}{78,160620} & \\
\hline Case/control & $393 / 347$ & & & & & \\
\hline Model $1^{*}$ & 1.00 & 0.82 & $0.68,1.01$ & 0.77 & $0.63,0.94$ & 0.039 \\
\hline Model $2 \dagger$ & 1.00 & 1.00 & $0.77,1.31$ & 0.92 & $0.70,1.21$ & 0.74 \\
\hline \multicolumn{7}{|l|}{ Dihydrodaidzein } \\
\hline Median & 2 & \multicolumn{2}{|c|}{38} & \multicolumn{2}{|c|}{568} & \\
\hline Range & 0,14 & \multicolumn{2}{|c|}{14,126} & & & \\
\hline Case/control & $392 / 348$ & & & & & \\
\hline Model $1^{*}$ & 1.00 & 0.90 & $0.73,1.10$ & 0.78 & $0.63,0.97$ & 0.043 \\
\hline Model $2 \dagger$ & 1.00 & 0.94 & $0.72,1.23$ & 0.80 & $0.60,1.06$ & 0.16 \\
\hline Dihydrogenistein & & & & & & \\
\hline Median & 7 & & & & & \\
\hline Range & 0,15 & & & & & \\
\hline Case/control & $389 / 351$ & & & & & \\
\hline Model $1^{*}$ & 1.00 & 0.84 & $0.68,1.02$ & 0.89 & $0.72,1.09$ & 0.55 \\
\hline Model $2 \dagger$ & 1.00 & 0.73 & $0.56,0.96$ & 0.82 & $0.62,1.08$ & 0.45 \\
\hline Total isoflavones & & & & & & \\
\hline Median & 198 & & & & & \\
\hline Range & 3,383 & & & & 500 & \\
\hline Case/control & $379 / 361$ & & & & & \\
\hline Model $1^{*}$ & 1.00 & 0.95 & $0.77,1.16$ & 0.92 & $0.75,1.13$ & 0.50 \\
\hline Model 2† & 1.00 & 0.83 & $0.63,1.08$ & 0.80 & $0.61,1.05$ & 0.20 \\
\hline
\end{tabular}

NHS, Nurses' Health Study.

* Model 1: conditional logistic model stratified by matching factors, including age at urine sample collection, month of sample collection, first morning urine (yes or no) and race (White or other races) in both cohorts. Menopausal status (pre-menopausal or post-menopausal), and hormone replacement therapy (yes or no) were additionally matched for in NHSII.

† Model 2: conditional logistic model were additionally adjusted for family history of diabetes (yes or no), hypertension at baseline (yes or no), hypercholesterolaemia at baseline (yes or no), BMI $\left(\mathrm{kg} / \mathrm{m}^{2}\right)$, smoking (non-smoker, past smoker, current smoker), alternative healthy eating index, physical activity (MET-h/week), total energy intake (kJ/d (kcal/d)), menopausal status (pre-menopausal or post-menopausal) and hormone replacement therapy (yes or no).

was found in a Chinese cohort ${ }^{(41)}$, although a positive association was found in the Multi-ethnic Cohort in Hawaii ${ }^{(42)}$. Differences in processing and cooking methods of soya food might be an explanation for these inconsistent associations. Moreover, measurement errors of FFQ assessments may be of a particular concern, especially among Western populations who consume much less soya foods than do Asians. To our knowledge, no previous prospective studies have examined isoflavone biomarkers in relation to T2D risk. In cross-sectional studies on the association between urinary isoflavones and blood glucose concentration, no associations were found ${ }^{(43)}$. The lack of association might be because of the reverse causation bias that the diabetes participants might tend to increase consumption of plant-based diet containing soya foods.
Our study had several strengths, including large sample size, long follow-up duration and use of urinary biomarkers to represent the internal dose of isoflavones and to account for the inter-individual variations in bioavailability. Our study also had several limitations. First, as reflected by the low ICC of isoflavone markers in spot urine samples, the urinary biomarkers might not represent long-term excretion of isoflavones well. The low ICC might result from the short half-lives of isoflavone metabolites in the human body, low frequency of consumption, and use of spot urine samples with poor time integration that were collected at various time points after the last meal. In general, non-differential misclassification of the exposure biases the results towards null. Moreover, because of the low reproducibility as well as the measurement errors associated with FFQ assessments of soya foods, only moderate correlation was 
Table 3. Stratified analysis of the association between urine isoflavones biomarkers and risk of type 2 diabetes by menopausal status and postmenopausal hormone use in the combined cohort*

(Odds ratios and $95 \%$ confidence intervals)

\begin{tabular}{|c|c|c|c|c|c|c|c|}
\hline & \multirow[b]{2}{*}{ Number of participants } & \multirow[b]{2}{*}{$\mathrm{T} 1$} & \multicolumn{2}{|c|}{ T2 } & \multicolumn{2}{|c|}{ T3 } & \multirow[b]{2}{*}{$P_{\text {interaction }} \uparrow$} \\
\hline & & & OR & $95 \% \mathrm{Cl}$ & OR & $95 \% \mathrm{Cl}$ & \\
\hline \multicolumn{8}{|l|}{ Daidzein } \\
\hline Pre-menopausal & 975 & 1.00 & 0.85 & $0.58,1.24$ & 0.81 & $0.54,1.21$ & \\
\hline Post-menopausal with HRT & 821 & 1.00 & 0.86 & $0.59,1.25$ & 0.76 & $0.52,1.11$ & \\
\hline Post-menopausal without HRT & 426 & 1.00 & 0.70 & $0.41,1.19$ & 0.58 & $0.34,0.97$ & 0.001 \\
\hline \multicolumn{8}{|l|}{ Genistein } \\
\hline Pre-menopausal & 975 & 1.00 & 0.96 & $0.66,1.41$ & 1.03 & $0.70,1.52$ & \\
\hline Post-menopausal with HRT & 821 & 1.00 & 0.73 & $0.50,1.07$ & 0.75 & $0.52,1.10$ & \\
\hline Post-menopausal without HRT & 426 & 1.00 & 0.58 & $0.34,1.00$ & 0.51 & $0.30,0.86$ & 0.36 \\
\hline \multicolumn{8}{|l|}{ DMA } \\
\hline Pre-menopausal & 975 & 1.00 & $1 \cdot 19$ & $0.80,1.77$ & 1.27 & $0.86,1.87$ & \\
\hline Post-menopausal with HRT & 821 & 1.00 & 1.06 & $0.73,1.53$ & 0.74 & $0.51,1.09$ & \\
\hline Post-menopausal without HRT & 426 & 1.00 & 0.79 & $0.47,1.34$ & 0.77 & $0.45,1.33$ & 0.21 \\
\hline \multicolumn{8}{|l|}{ DHDE } \\
\hline Pre-menopausal & 975 & 1.00 & 1.22 & $0.82,1.80$ & $1 \cdot 22$ & $0.82,1.82$ & \\
\hline Post-menopausal with HRT & 821 & 1.00 & 0.99 & $0.68,1.43$ & 0.65 & $0.44,0.96$ & \\
\hline Post-menopausal without HRT & 426 & 1.00 & 0.75 & $0.44,1.28$ & 0.63 & $0.37,1.05$ & 0.82 \\
\hline \multicolumn{8}{|l|}{ DHGE } \\
\hline Pre-menopausal & 975 & 1.00 & 0.70 & $0.48,1.03$ & 1.03 & $0.70,1.51$ & \\
\hline Post-menopausal with HRT & 821 & 1.00 & 0.84 & $0.57,1.23$ & 0.66 & $0.45,0.96$ & \\
\hline Post-menopausal without HRT & 426 & 1.00 & 0.80 & $0.47,1.36$ & 0.71 & $0.42,1.22$ & 0.90 \\
\hline
\end{tabular}

DHDE, dihydrodaidzein; DHGE, dihydrogenistein; DMA, desmethylangolensin; HRT, hormone replacement therapy; NHS, Nurses' Health Study; T, tertile.

* Given that menopausal status and HRT were not matching factors in NHS, unconditional logistic models were used for the stratified analysis and multivariate models adjusted for age at urine sample collection, month of sample collection, fasting status ( $\geq 8 \mathrm{~h}$ or not), first morning urine (yes or no), race (White or other races), family history of diabetes (yes or no), hypertension at baseline (yes or no), hypercholesterolaemia at baseline (yes or no), BMI (continuous), smoking (non-smoker, past smoker, current smoker), alternative healthy eating index (continuous), physical activity (continuous) and total energy intake (continuous).

† Likelihood ratio test comparing the conditional logistic models with and without interaction term was used; the urine isoflavones were dichotomised and the pre-menopausal and post-menopausal with HRT were combined into one group.

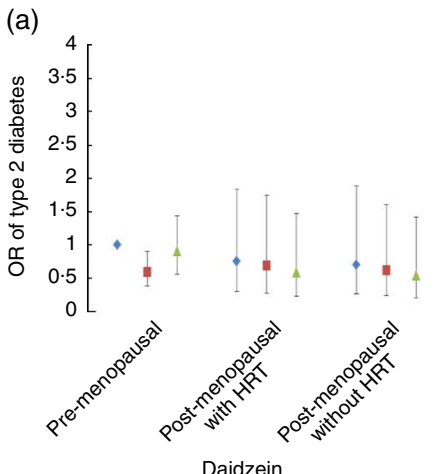

(d)

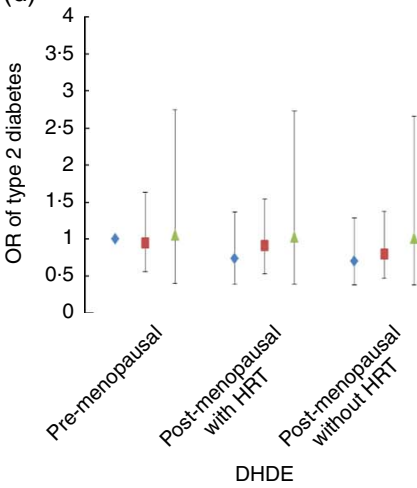

(b)
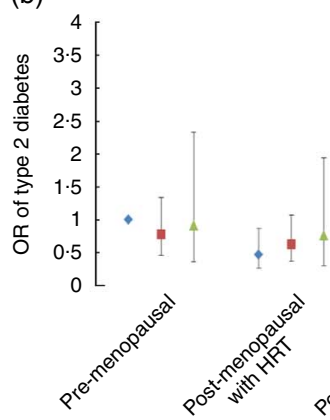

Genistein

(e)

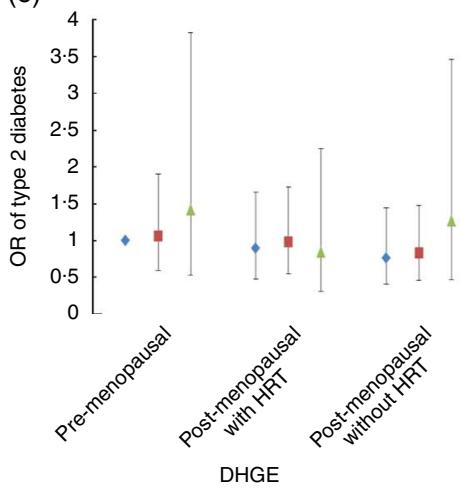

(c)

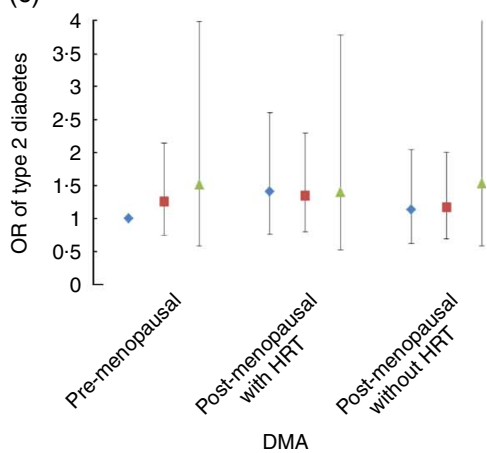

Fig. 1. Joint association of urinary isoflavone biomarkers and post-menopausal status and hormone use with odds of type 2 diabetes. Conditional logistic models are adjusted for hypertension at baseline (yes or no), hypercholesterolaemia at baseline (yes or no), BMI $\left(\mathrm{kg} / \mathrm{m}^{2}\right)$, smoking (non-smoker, past smoker, current smoker), alternative healthy eating index, physical activity (MET-h/week) and total energy intake (kJ/d (kcal/d)). DHDE, dihydrodaidzein; DHGE, dihydrogenistein; DMA, desmethylangolensin; HRT, hormone replacement therapy. $\diamond$, Lowest tertile; $\square$, second lowest tertile; $\triangle$, highest tertile. 
found between soya foods assessed by FFQ and urinary isoflavones, indicating that urinary isoflavones might not represent long-term relatively low soya food consumption well. Meanwhile, given the between-person variability in isoflavones' bioavailability partially due to microbiota processing and other physiological processes during absorption and metabolism ${ }^{(44)}$, FFQ assessments of isoflavones and soya foods may not measure exposures to these isoflavone metabolites well. Second, although we adjusted for an array of established and potential risk factors of $\mathrm{T} 2 \mathrm{D}$, we could not exclude the possibility that un-measured confounding or residual confounding, such as a healthy lifestyle, might still partially explain the association between isoflavones and risk of T2D. Third, we cannot exclude the possibility that chance may play a role in our findings, especially when the inverse association was primarily observed for daidzein. Fourth, we did not observe significant association between total urinary isoflavones and risk of T2D. Given that these individual metabolites have different levels of measurement errors, different time integration (as reflected by ICC) and different levels of microbiota processing, the associations for total isoflavones may be prone to attenuation towards the null due to this heterogeneity. Lastly, the observed associations may have limited generalisability within White health professionals. In addition, the urinary excretion of isoflavones was somehow lower than that was observed in NHANES (mean urinary isoflavones: $4.9 v \cdot 149 \cdot 1 \mu \mathrm{g} / \mathrm{g}$ creatine in our study), further limiting the generalisability to populations with high levels of isoflavone intake.

In conclusion, we observed inverse associations between urinary excretion of daidzein and genistein and risk of T2D in US women. In addition, the inverse association for daidzein was stronger among post-menopausal women who did not use hormone replacement therapy. Although these findings are in line with evidence from animal experiments and clinical trials demonstrating benefits of isoflavone intake on insulin resistance, further studies are warranted to replicate the current findings among other western populations with low isoflavone intake levels. In addition, multiple-day $24 \mathrm{~h}$ urine samples shall be used to achieve more stable estimates of isoflavone-marker excretion.

\section{Acknowledgements}

The authors acknowledge Mary Kay Townsend and Kimberly A. Bertrand's work on conducting pilot studies for the current investigation.

R. Mv. D. and F. B. H. obtained funding from the National Institutes of Health. This study was funded by research grants CA186107, CA49449, CA176726, CA67262, DK58845, DK58785, DK082486, CA50385, CA87969 and P30 CA71789 from the National Institutes of Health. Dr Sun was supported by a career development award R00HL098459 from the National Heart, Lung and Blood Institute. The funding sources had no role in any of the following: in study design; in the collection, analysis and interpretation of data; in the writing of the report; or in the decision to submit the article for publication. The authors are not affiliated with the funding institutions.

Q. S., A. A. F., F. B. H. and R. Mv. D. were involved in data collection of dietary flavonoids or urinary metabolites. A. A. F. measured urinary metabolites using liquid chromatography MS. Q. S. and R. Mv. D. conducted pilot studies for the current investigation. Q. S., F. B. H., B. A. R. and R. Mv. D. provided statistical expertise. M. D. analysed the data and wrote the first draft of the manuscript. Q. S., F. B. H., A. A. F., S. S. T., B. A. R. and R. Mv. D. contributed to the interpretation of the results and critical revision of the manuscript for important intellectual content and approved the final version of the manuscript. Q. S. is the guarantor of this investigation.

None of the authors has any financial or personal conflicts of interest to disclose.

\section{Supplementary material}

To view supplementary material for this article, please visit http://dx.doi.org/10.1017/S0007114515003359

\section{References}

1. International Diabetes Federation (2013) International Diabetes Federation. IDF Diabetes Atlas, 6th ed. Brussels, Belgium: International Diabetes Federation.

2. Hu FB, Manson JE, Stampfer MJ, et al. (2001) Diet, lifestyle, and the risk of type 2 diabetes mellitus in women. $N$ Engl J Med 345, 790-797.

3. Fung TT, Schulze M, Manson JE, et al. (2004) Dietary patterns, meat intake, and the risk of type 2 diabetes in women. Arch Intern Med 164, 2235-2240.

4. Van Dam RM, Rimm EB, Willett WC, et al. (2002) Dietary patterns and risk for type 2 diabetes mellitus in U.S. men. Ann Intern Med 136, 201-209.

5. Mozaffarian D, Hao T, Rimm EB, et al. (2011) Changes in diet and lifestyle and long-term weight gain in women and men. $N$ Engl J Med 364, 2392-2404.

6. Heianza Y, Arase Y, Kodama S, et al. (2013) Effect of postmenopausal status and age at menopause on type 2 diabetes and prediabetes in Japanese individuals: Toranomon Hospital Health Management Center Study 17 (TOPICS 17). Diabetes Care 36, 4007-4014.

7. Szmuilowicz ED, Stuenkel CA \& Seely EW (2009) Influence of menopause on diabetes and diabetes risk. Nat Rev Endocrinol 5, 553-558.

8. Cho GJ, Lee JH, Park HT, et al. (2008) Postmenopausal status according to years since menopause as an independent risk factor for the metabolic syndrome. Menopause 15, 524-529.

9. Kanaya AM, Herrington D, Vittinghoff E, et al. (2003) Glycemic effects of postmenopausal hormone therapy: the Heart and Estrogen/progestin Replacement Study. A randomized, doubleblind, placebo-controlled trial. Ann Intern Med 138, 1-9.

10. Manson JE, Chlebowski RT, Stefanick ML, et al. (2013) Menopausal hormone therapy and health outcomes during the intervention and extended poststopping phases of the Women's Health Initiative randomized trials. J Am Med Assoc 310, 1353-1368.

11. Manson JE, Rimm EB, Colditz GA, et al. (1992) A prospective study of postmenopausal estrogen therapy and subsequent incidence of non-insulin-dependent diabetes mellitus. Ann Epidemiol 2, 665-673.

12. Rossi R, Origliani G \& Modena MG (2004) Transdermal 17-beta-estradiol and risk of developing type 2 diabetes in a population of healthy, nonobese postmenopausal women. Diabetes Care 27, 645-649.

13. Liu ZM, Chen YM \& Ho SC (2011) Effects of soy intake on glycemic control: a meta-analysis of randomized controlled trials. Am J Clin Nutr 93, 1092-1101. 
14. Yang B, Chen $\mathrm{Y}$, Xu T, et al. (2011) Systematic review and meta-analysis of soy products consumption in patients with type 2 diabetes mellitus. Asia Pac J Clin Nutr 20 593-602.

15. Mueller NT, Odegaard AO, Gross MD, et al. (2012) Soy intake and risk of type 2 diabetes in Chinese Singaporeans [corrected]. Eur J Nutr 51, 1033-1040.

16. Nanri A, Mizoue T, Takahashi Y, et al. (2010) Soy product and isoflavone intakes are associated with a lower risk of type 2 diabetes in overweight Japanese women. J Nutr $\mathbf{1 4 0}$, 580-586.

17. Zamora-Ros R, Forouhi NG, Sharp SJ, et al. (2013) The association between dietary flavonoid and lignan intakes and incident type 2 diabetes in European populations: the EPIC-InterAct study. Diabetes Care 36, 3961-3970.

18. Bhagwat S, Haytowitz DB \& Holden JM (2008) USDA database for the isoflavone content of selected foods release 2.0.

19. Spencer JP, Abd El Mohsen MM, Minihane AM, et al. (2008) Biomarkers of the intake of dietary polyphenols: strengths, limitations and application in nutrition research. BrJ Nutr 99, $12-22$.

20. Zamora-Ros R, Touillaud M, Rothwell JA, et al. (2014) Measuring exposure to the polyphenol metabolome in observational epidemiologic studies: current tools and applications and their limits. Am J Clin Nutr 100, 11-26.

21. Franke AA, Custer LJ, Wilkens LR, et al. (2002) Liquid chromatographic-photodiode array mass spectrometric analysis of dietary phytoestrogens from human urine and blood. J Chromatogr B Analyt Technol Biomed Life Sci 777, 45-59.

22. Perez-Jimenez J, Hubert J, Hooper L, et al. (2010) Urinary metabolites as biomarkers of polyphenol intake in humans: a systematic review. Am J Clin Nutr 92, 801-809.

23. Muthyala RS, Ju YH, Sheng S, et al. (2004) Equol, a natural estrogenic metabolite from soy isoflavones: convenient preparation and resolution of $\mathrm{R}$ - and S-equols and their differing binding and biological activity through estrogen receptors alpha and beta. Bioorg Med Chem 12, 1559-1567.

24. Seow A, Shi CY, Franke AA, et al. (1998) Isoflavonoid levels in spot urine are associated with frequency of dietary soy intake in a population-based sample of middle-aged and older Chinese in Singapore. Cancer Epidemiol Biomarkers Prev 7, 135-140.

25. Maskarinec G, Singh S, Meng L, et al. (1998) Dietary soy intake and urinary isoflavone excretion among women from a multiethnic population. Cancer Epidemiol Biomarkers Prev 7, 613-619.

26. Prentice RL \& Breslow NE (1978) Retrospective studies and failure time models. Biometrika 65, 153-158.

27. Manson JE, Rimm EB, Stampfer MJ, et al. (1991) Physical activity and incidence of non-insulin-dependent diabetes mellitus in women. Lancet 338, 774-778.

28. Willett WC (1998) Nutritional Epidemiology, 2nd ed. New York: Oxford University Press.
29. McCullough ML \& Willett WC (2006) Evaluating adherence to recommended diets in adults: the Alternate Healthy Eating Index. Public Health Nutr 9, 152-157.

30. Chiuve SE, Fung TT, Rimm EB, et al. (2012) Alternative dietary indices both strongly predict risk of chronic disease. $J$ Nutr 142, 1009-1018.

31. Franke AA, Halm BM, Kakazu K, et al. (2009) Phytoestrogenic isoflavonoids in epidemiologic and clinical research. Drug Test Anal 1, 14-21.

32. Messina MJ \& Wood CE (2008) Soy isoflavones, estrogen therapy, and breast cancer risk: analysis and commentary. Nutr J 7, 17.

33. Ricci E, Cipriani S, Chiaffarino F, et al. (2010) Effects of soy isoflavones and genistein on glucose metabolism in perimenopausal and postmenopausal non-Asian women: A meta-analysis of randomized controlled trials. Menopause 17, 1080-1086.

34. Golditz GA (1998) Relationship between estrogen levels, use of hormone replacement therapy, and breast cancer. $J$ Natl Cancer Inst 90, 814-823.

35. Maccio A \& Madeddu C (2011) Obesity, inflammation, and postmenopausal breast cancer: therapeutic implications. Sci World J 11, 2020-2036.

36. Mezei O, Banz WJ, Steger RW, et al. (2003) Soy isoflavones exert antidiabetic and hypolipidemic effects through the PPAR pathways in obese Zucker rats and murine RAW 264.7 cells. J Nutr 133, 1238-1243.

37. Kim S, Sohn I, Ahn JI, et al. (2004) Hepatic gene expression profiles in a long-term high-fat diet-induced obesity mouse model. Gene 340, 99-109.

38. Mezei O, Li Y, Mullen E, et al. (2006) Dietary isoflavone supplementation modulates lipid metabolism via PPARalphadependent and -independent mechanisms. Physiol Genomics 26, 8-14.

39. Cederroth CR, Vinciguerra M, Gjinovci A, et al. (2008) Dietary phytoestrogens activate AMP-activated protein kinase with improvement in lipid and glucose metabolism. Diabetes $\mathbf{5 7}$, 1176-1185.

40. Babu PV, Liu D \& Gilbert ER (2013) Recent advances in understanding the anti-diabetic actions of dietary flavonoids. J Nutr Biochem 24, 1777-1789.

41. Villegas R, Gao YT, Yang G, et al. (2008) Legume and soy food intake and the incidence of type 2 diabetes in the Shanghai Women's Health Study. Am J Clin Nutr 87, 162-167.

42. Morimoto Y, Steinbrecher A, Kolonel LN, et al. (2011) Soy consumption is not protective against diabetes in Hawaii: the Multiethnic Cohort. Eur J Clin Nutr 65, 279-282.

43. Struja T, Richard A, Linseisen J, et al. (2014) The association between urinary phytoestrogen excretion and components of the metabolic syndrome in NHANES. Eur J Nutr 53, 1371-1381.

44. Rowland IR, Wiseman H, Sanders TA, et al. (2000) Interindividual variation in metabolism of soy isoflavones and lignans: influence of habitual diet on equol production by the gut microflora. Nutr Cancer 36, 27-32. 